\title{
In-situ TEM Experiments to Assess the Predictive Capability of Atomistic models
}

\author{
H. D. Espinosa,* B. Peng, R. Agrawal, R. A. Bernal
}

Department of Mechanical Engineering, Northwestern University, 2145 Sheridan Road, Evanston, IL 60208-3111

In-situ microscopy testing empowered with the versatility of microelectromechanical systems (MEMS) has played a vital role in unveiling structure-property relationships. We developed a MEMS-based nanoscale-Material Testing system (n-MTS) [1-2], which interfaces with electron microscopes to perform in situ mechanical and electromechanical testing of one-dimensional nanostructures. In this set-up, the quantitative load-displacement data is acquired electronically and the specimen is monitored with atomic-level resolution during the test. Uniaxial loading condition is maintained which facilitates data interpretation with minimal assumptions. This unique set-up was used to investigate carbon nanotubes (CNTs) and semiconducting nanowires (NWs), in turn providing an assessment of existing predictive capabilities of the atomistic models.

In-situ TEM, multi-walled carbon nanotubes (MWNTs) subjected to various degrees of electron and ion irradiation were mechanically tested [3-4]. We observed that below a threshold radiation, the MWNTs failed by a telescopic or "sword-in-sheath" mechanism. Analysis of the high resolution TEM images clearly revealed the number of shells fractured. For an experiment, where just one shell failed, a Young's modulus of $998 \mathrm{GPa}$ and a failure stress of $97 \mathrm{GPa}$ were measured, which is in good agreement with values predicted by Density Functional Theory (DFT) calculations for pristine single-walled CNTs. These results were the first of their kind allowing for a direct comparison with the theoretical predictions, validating the approximations involved in modeling. In addition to mechanical characterization of MWNTs, elasticity size effects and failure mechanisms in zinc oxide $(\mathrm{ZnO}) \mathrm{NWs}$ were also investigated using a combined experimental-computational approach. In-situ TEM experiments resolved the discrepancy concerning size dependent elastic modulus of $\mathrm{ZnO}$ NWs, revealing increasing elastic modulus with decreasing wire diameter [5]. Molecular dynamics (MD) simulations using a Buckingham type potential predicted a trend consistent with the experimental findings. However, the failure mechanisms observed in experiments and simulations were different. The experiments revealed brittle fracture whereas MD simulations predicted a phase transformation, which casted doubts on the accuracy of Buckingham type potential in capturing the phenomena of fracture [6]. Therefore, first-principles based calculations are being pursued to validate the accuracy of the MD force fields for predicting failure modes. We are also involved in characterizing the electromechanical coupling and measuring the piezoelectric coefficients in gallium nitride nanowires as a function of their characteristic size. Preliminary results suggest there might be an enhancement in piezoelectric properties of smaller nanowires.

\section{References}

[1] Espinosa, H.D., Zhu, Y., Moldovan, N., Journal of Microelectromechanical Systems, 16 (2007) 5 p.1219-1231.

[2] Y. Zhu, and H.D. Espinosa, Proceedings of the National Academy of Sciences of the United States of America, 102 (2005) 41 p. 14503-14508.

[3] Peng, B., et al., Nature Nanotechnology, 3 (2008) 10 p. 626 - 631.

[4] Locascio, M., et al., Experimental Mechanics, 49 (2009) 2 p. 169-182. 
[5] Agrawal, R., et al., Nano Letters, 8 (2008) 11 p. 3668-3674.

[6] Agrawal, R., Peng, B., Espinosa, H. D., Nano Letters, 9 (2009) 12 p. 4177-4183.

[7] This research was supoprted by the National Science Foundation through Awards No. CMMI -0555734, DMR-0907196, and EEC - 0647560. The support from ONR through Award No. N00014-08-1-0108 and ARO through Awards No. W911NF-08-1-0541 and W911NF-08-10061 is also greatly appreciated. We also thank I. Petrov and E. Olson for their contribution towards the manufacturing of the in situ TEM holder used in the work here reported. We acknowledge N. Sanford and K. Bartess, from the National Institute of Standards and Technology, for providing GaN nanowires. 